Research Article

\title{
Cooperative Diversity in Wireless Networks
}

\author{
A. Mahmood ${ }^{*}$ \\ School of Information Science \& Engineering, Shandong Province, People’s Republic of China.
}

Received 6 February 2010; Revised 20 July 2010; Accepted 29 August 2010

\begin{abstract}
Transmit Diversity is an effective methodology for improving the quality and reliability of a wireless network by reducing the effects of fading. As majority of the wireless devices (i.e. mobile handsets, etc) are limited to only one antenna, especially due to hardware constraints, size and cost factors; cooperative communication can be utilized in order to generate transmit diversity [1]. This enables single antenna wireless devices to share their antennas during transmission in such a manner that creates a virtual MIMO (multiple-input and multiple-output) system [2] [3]. In this paper, we will analyze the recent developments and trends in this promising area of wireless Ad hoc networks. The article will also discuss various main cooperative signaling methods and will also observe their performance.
\end{abstract}

Keywords: Cooperative Communication, Multiple Input Multiple Output Systems, Transmit Diversity, Adhoc Networks etc.

\section{Introduction}

"The increasing demand for wireless multimedia and interactive Internet services, along with rapid proliferation of a multitude of communications and computational gadgets, are fuelling intensive research efforts on the design of novel wireless communication systems architectures for high speed, reliable and cost effective transmission solutions" [4]. The introduction and rapid development of MIMO (multiple-input and multiple-output) systems has promised significant improvements in reliability and throughput for Ad hoc networks; by utilizing multiple antennas at both the transmitter and the receive side. However, this technique is clearly advantageous for cellular base stations, but not feasible for mobile devices, due to their sizes and power constraints [1] [4].

An alternate to this is a newly developed technique known as multi-user cooperative diversity that allows a single antenna user to achieve transmit diversity benefits by sharing their physical resources through a virtual transmit and receive antenna array. The major benefit of this technique includes the diversity - because different paths are likely to fade independently, beamforming gain and interference mitigation [1] [5].

\section{Cooperative Communication}

The history of the cooperative communication can found its deep roots to the groundbreaking work of Van der Meulen [6], when he introduced the concept of relay channel model, which consists of a source, destination and relay; and whose major purpose was to facilitate the information transfer from source to destination. Later, Cover and El Gamal [7] deeply investigated the relay channel model, and provided a number of fundamental relaying techniques such as Decode and Forward (DF) and Compress and Forward (CF).

In conventional communication, data is transmitted between the source and destination, and no user provides assistance to one another (Figure 1). However, there are many neighboring nodes in a practical wireless communication network, which could be of great assistance. When one node transmits its data, all the nearby nodes overheard its transmission. Cooperative Communication aims to process and forward this overhead information to the respective destination to create spatial diversity, which can in result increase

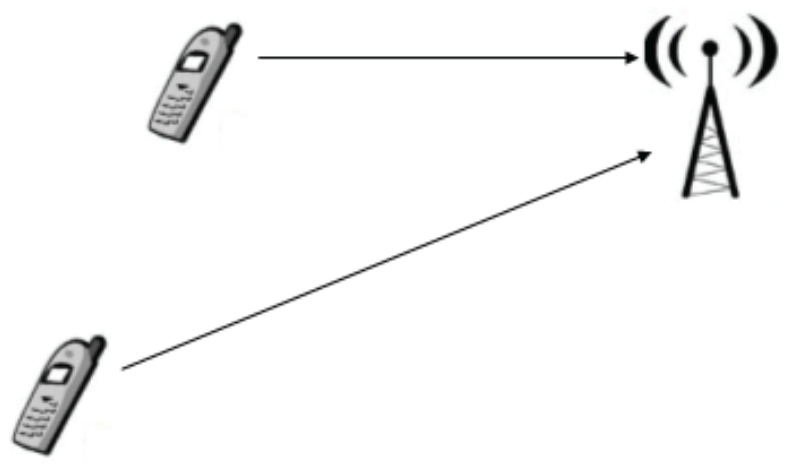

Figure 1. Conventional Communication. 
the system performance. The concept of the cooperative communication is suggested in Figure 2. [4]

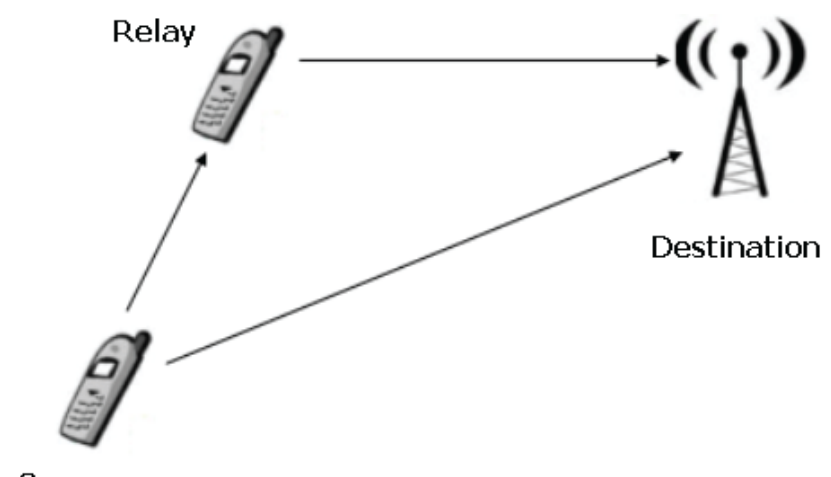

Source

Figure 2. Cooperative Communication.

As depicted in Figure 2, the source ' $\mathrm{S}$ ' is transmitting data to the destination ' $\mathrm{D}$ ', while the relay station (another mobile user) ' $R$ ' is also hearing the transmission. The relay station also process and forward this message to the destination, where both of the received signals are combined. As both copies of the signals are transmitted through independent paths, this results into spatial diversity. In cooperative communication, each wireless user is assumed to transmit its own data as well as act as a cooperative agent (relay) for the other user (Figure 3). [1] [8]

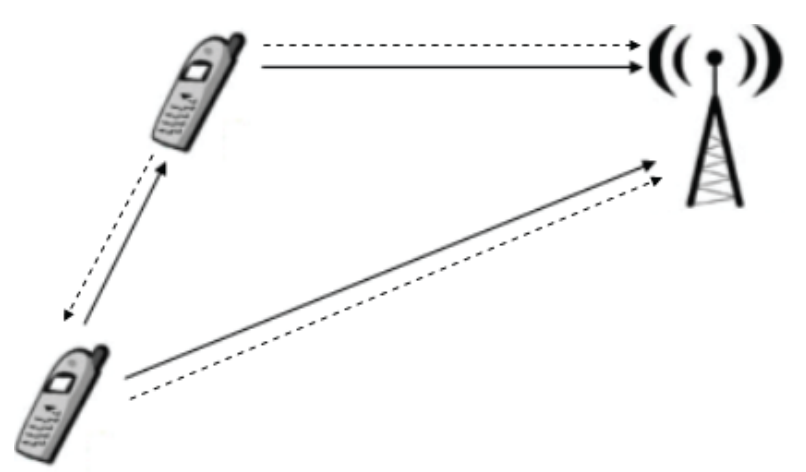

Figure 3. Cooperative Communication, where each mobile act both as a user and relay.

\section{Cooperative Transmission Protocols}

Cooperative transmission protocols describes that how the received data is processed at the relay station, before forwarding it to the destination. In this article, we will review and compare several cooperative strategies in brief.

\section{A. Decode and Forward}

The Decode and Forward (DF) method is the most preferred method of processing data in the relay/partners/neighboring nodes, and is closest to the idea of a traditional relay. In this technique, the relay detects the source data, decodes and then transmits it to the desired destination. The concept of the Decode and Forward technique is shown in Figure 4.

\section{Decode and Forward}

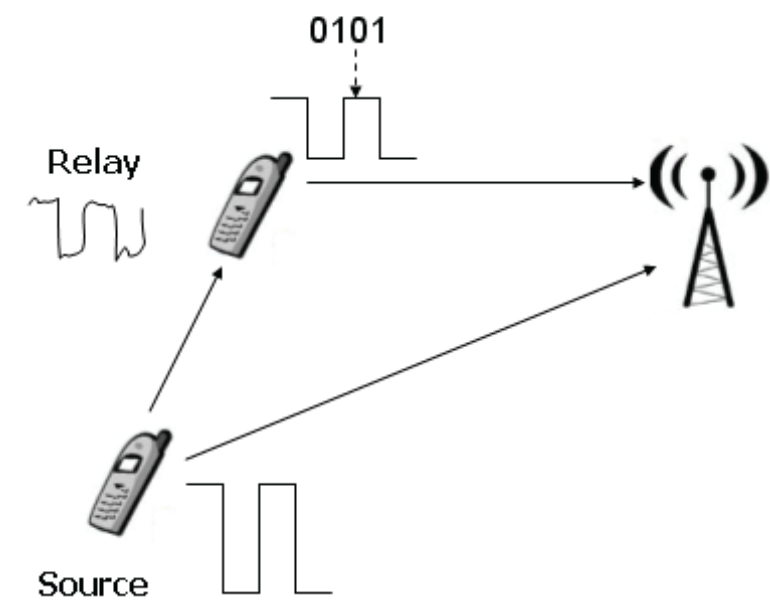

Figure 4. Decode and Forward Technique.

An error correcting code can also be implemented at the relay station. This could help the received bit errors to be corrected at the relay station. However, this is only possible, if the relay station has enough computing power [8].

\section{B. Amplify and Forward}

As the name suggests, the Amplify and Forward technique simply amplify the signal received by the relay before forwarding it to the destination. This technique was proposed by J. N. Laneman and G. W. Wornell [9], and is ideal when the relay station has minimal computing power. However, one major drawback of this technique is that the noise in the signal is also amplified at the relay station, and the destination receives two independently faded versions of the signal. The concept of the Decode and Forward technique is shown in Figure 5. The interested reader is referred to literature of J. N. Laneman and G. W. Wornell [9] for a comprehensive understanding of the Amplify and Forward technique.

\section{Amplify and Forward}

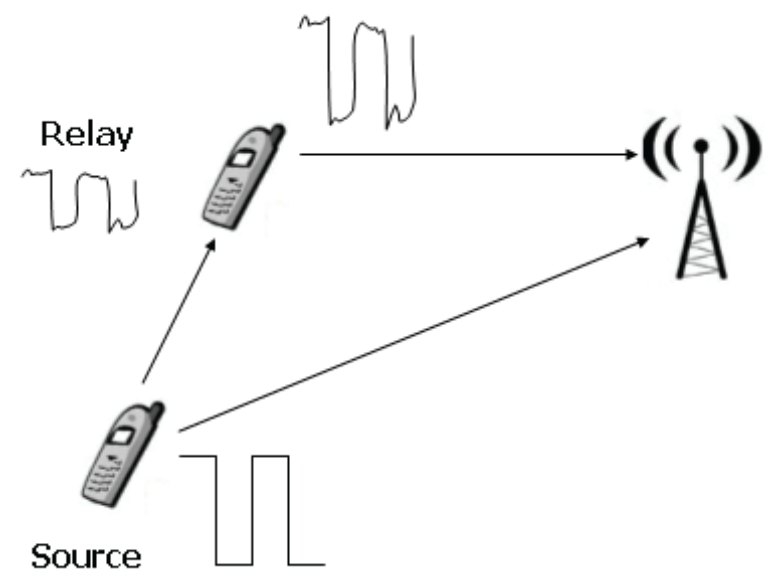

Figure 5. Amplify and Forward Technique. 


\section{Coded Cooperation}

Todd E. Hunter and Aria Nosratinia [10] [11] proposed a new technique known as coded cooperation. In the other two cooperative strategies described above, the partner / relay station just retransmits the received data bits by decoding (DF Method) or amplifying (AF Method) [3]. Unlike these two techniques, the received data bits in coded cooperation are broken down into two parts. One part is transmitted by the user and the other part is transmitted by the partner. The concept of the two user coded cooperation system is shown in Figure 6. As depicted in the figure, the User 1 is transmitting its own half data bits in Frame 1, and the partner (User 2) half data bits in Frame 2 to the destination. However, in actual wireless cellular network, there could be more than 2 cooperative users and data transmitted by one user can be overheard by other users as well (Figure 7). Salam A Zummo [3] proposed an analytical framework for deriving and evaluating the error performance of coded cooperation with multiple cooperating users.

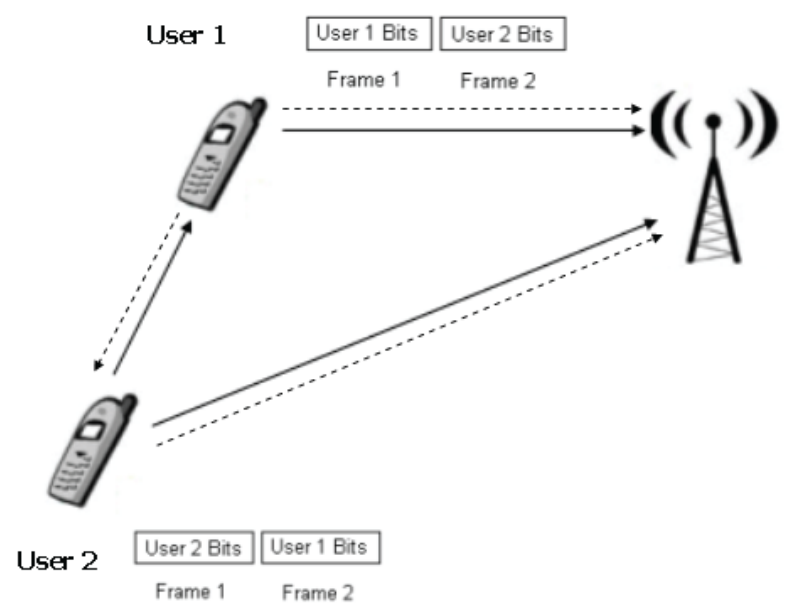

Figure 6. Coded Cooperation for 2 Users.

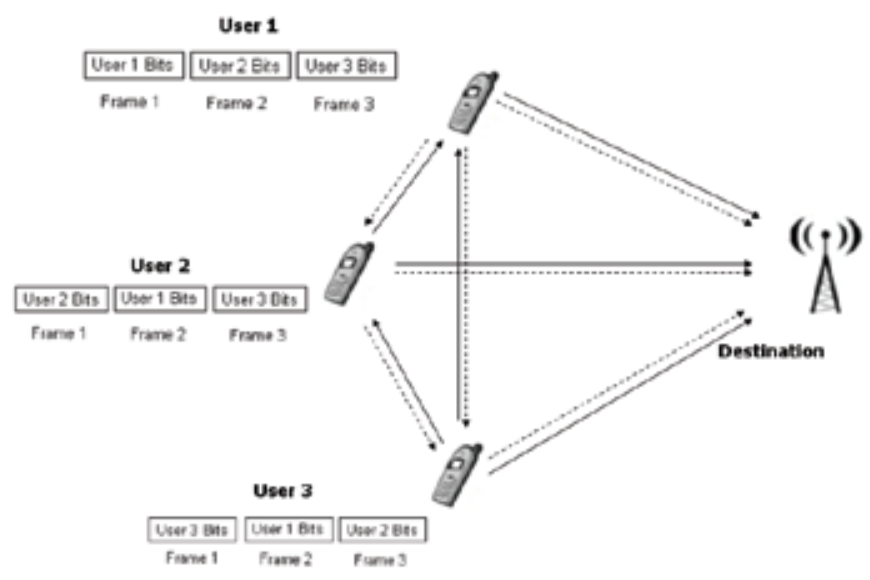

Figure 7. Coded Cooperation 3 Users.

\section{Performance Analysis}

Figure 8 provides the performance analysis of the already discussed cooperative strategies; Decode and Forward, Amplify and Forward, and Coded Cooperation using BPSK modulation and coherent detection at the receiver. The comparison clearly suggests that the coded cooperation technique performs better than the Decode and Forward and Amplify and Forward techniques, since it has better degradation. The graph also suggests that the error performance rate has substantially improved as a result of cooperative communication between users [1].

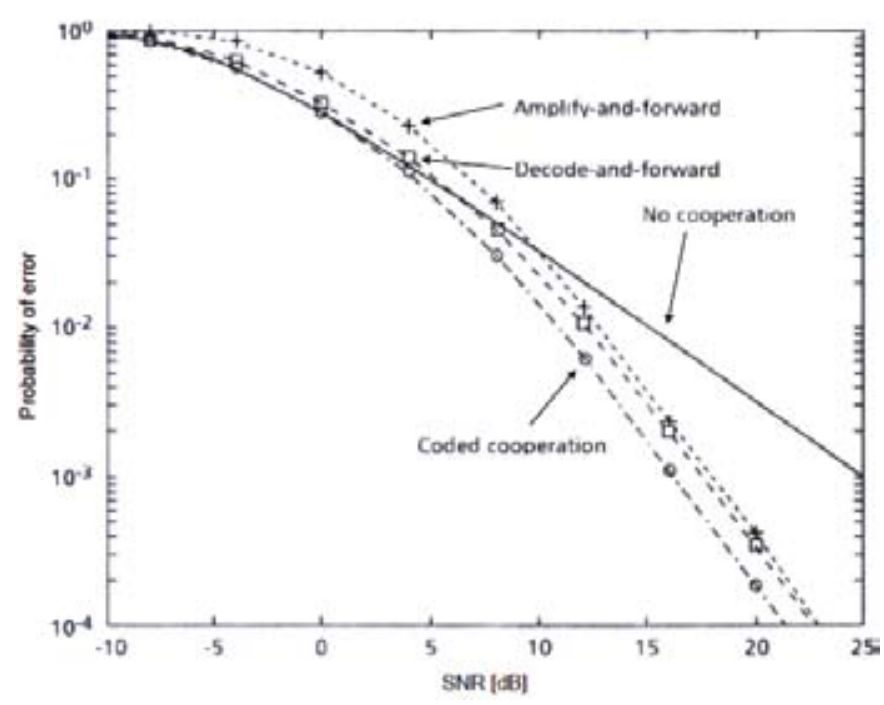

Figure 8. Performance Analysis.

\section{Acknowledgment}

The author would like to express his sincere appreciation to $\mathrm{Mr}$. Yang Xinghai (Associate Professor and Supervisor) for providing his valuable guidance, technical inputs and advice throughout this research. Above all, he has provided me persistent encouragement and support in various ways. His truly scientist intuition has made him as a constant oasis of ideas and passions in science, which exceptionally inspire and enrich my growth as a student and as a researcher, and for this I am always indebted to him more than he himself knows. His insights and suggestions had helped us to greatly improve this paper. 


\section{References}

1. A. Nosratinia, T. E. Hunter, A. Hedayat, "Cooperative Communications in Wireless Networks", IEEE Communications Magazine, 2004, pp. 74-80.

2. K. Tahir, "Coded Cooperative Communications", MSc Thesis, Blekinge Institute of Technology (BTH), Sweden, September 2007

3. S. A. Zummo, "Performance Analysis of Coded Cooperation Diversity in Wireless Networks", Journal of Wireless Communications and Mobile Computing, John Wiley and Sons Ltd, 2005.

4. M. Uysal, "Cooperative Communications for Improved Wireless Network Transmission”, Information Science Reference, July 2009.

5. G. K. Karagiannidis, C. Tellambura, S. Mukherjee, A. O. Fapojuwo, "Editorial - Multi-user Cooperative Diversity for Wireless Networks", EURASIP Journal on Wireless Communications and Networking.

6. E. C. Van Der Meulen, "Three-terminal communication channels", Advances in Applied Probability, 3:120-154, 1971.

7. T. M. Cover and A. A. E. Gamal, "Capacity Theorems for the Relay Channel”, IEEE Trans. Info. Theory, vol. 25, no. 5, Sept. 1979, pp. 572-584.

8. A. Meier, "Cooperative Diversity in Wireless Networks", MSc Thesis, University of Edinburgh, 2004.

9. J. N. Laneman, G. W. Wornell and D. N. C. Tse, “An Efficient Protocol for Realizing Cooperative Diversity in Wireless Networks", Proc. IEEE ISIT, Washington, DC, June 2001, p. 294.

10. T. E. Hunter and A. Nosratinia, "Cooperative Diversity through Coding", Proc. IEEE ISIT, Laussane, Switzerland, July 2002, p. 220.

11. T. E. Hunter and A. Nosratinia, "Diversity through Coded Cooperation", submitted to IEEE Trans. Wireless Commun., 2004.

12. J. G. Proakis, "Digital Communications", McGraw Hill (4th Edition), 2001.

13. A. Papoulis, "Probability, Random Variables, and Stochastic Processes", McGraw Hill, 1965.

14. A. Viterbi, J. Omura, "Principles of Digital Communication and Coding", McGraw Hill, 1979.

15. Jakes W, "Microwave Mobile Communications", IEEE Press, 1974.

16. W. Stalling, "Wireless Communications and Networking", Pearson Education.

17. S. Haykin, "Digital Communication Systems", John Wiley and Sons Inc, 2003.

18. G. Kramer, M. Gastpar, "Cooperative Strategies and Capacity Theorems for Relay Networks”, IEEE Trans. Information Theory, vol. 51, no .9, September 2005.

19. M. Janani, A. Hedayat, T. E. Hunter and A. Nosratinia, "Coded cooperation in wireless communications: Space-time transmission and iterative decoding", IEEE Trans. Signal Processing, vol. 52, no. 2, pp. 362-371, Feb. 2004

20. A. del Coso, C. Ibars, "Capacity of decode-and-forward cooperative links with full channel state information", Proc. $39^{\text {th }}$ Asilomar Conference on Signals, Systems and Computers, Pacific Grove, CA, Nov. 4-6 2005.

21. T. M. Cover and A. A. E. Gamal, "Capacity Theorems for the Relay Channel”, IEEE Trans. Info. Theory", vol. 25, no. 5, Sept. 1979, pp. 572-84.

22. J. N. Laneman, D. Tse, "Cooperative diversity in wireless networks: efficient protocols and outage behaviour", IEEE Trans. Information Theory, vol. 50, no. 12, December 2004.

23. J. N. Laneman and G. W. Wornell, "Distributed space-time coded protocols for exploiting cooperative diversity in wireless networks", IEEE Trans. Inform. Theory, vol. 49, pp. 2415-2525, Oct. 2003.

24. R. U. Nabar and H. Bölcskei, "Fading relay channels: performance limits and Space-Time signal design", IEEE Journal on Selected Areas in Communications, Vol. 22, no. 6, August 2004.

25. B. Zhao and M. Valenti, "Some New Adaptive Protocols for the Wireless Relay Channel”, Proc. Allerton Conf. Commun., Control, and Comp., Monticello, IL, Oct. 2003.

26. P. Larsson, "Selection Diversity Forwarding in a Multihop Packet Radio Network with Fading Channel and Capture", Mobile Comp. Commun. Rev., vol. 5, no. 4, Oct. 2001, pp. 47-54.

27. S. Wei, D. L. Goeckel, M. C. Valenti, “Asynchronous cooperative diversity”, IEEE Trans. Wireless Commun., vol. 5, pp. 1547-1557, June 2006.

28. R. U. Nabar, F. W. Kneubuhler, H. Bölcskei, "Performance limits of amplify-and-forward based fading relay channels", IEEE Proc. ICASSP, vol. 4, pp. iv-565 - iv-568, May 2004.

29. Qian Zhang, Juncheng Jia, Jin Zhang, "Cooperative relay to improve diversity in cognitive radio networks," IEEE Communications Magazine, vol. 47, issue. 2, 2009, pp. 111-117.

30. Y. W Hong, W. J. Huang, F. H. Chiu, C. C. J. Kuo, "Cooperative Communications in Resource-Constrained Wireless Networks", IEEE Signal Processing Magazine, vol. 24, issue. 3, 2007, pp. 45-47.

31. A. Bletsas, A. Lippman, "Implementing cooperative diversity antenna arrays with commodity hardware", IEEE Communications Magazine, vol. 24, issue. 12, 2006, pp. 33-40.

32. A. Bletsas, A. Lippman, "Implementing cooperative diversity antenna arrays with commodity hardware", IEEE Communications Magazine, vol. 24, issue. 12, 2006, pp. 33-40.

33. R. Pabst, B. H. Walke, D. C. Schultz, P. Herhold, H. Yanikomeroglu, S Mukherjee, H. Viswanathan, M. Lott, W. Zirwas, M. Dohler, H. Aghvami, D. D. Falconer, G. P. Fettweis, "Relay-based deployment concepts for wireless and mobile broadband radio", IEEE Communications Magazine, vol. 42, issue. 09, 2004, pp. 80-89.

34. Yonghui Li, "Distributed coding for cooperative wireless networks: An overview and recent advances", IEEE Communications Magazine, vol. 47, issue. 08, 2009, pp. 71-77. 